\title{
Principal specialization of dual characters of flagged Weyl modules
}

\author{
Karola Mészáros* \\ Department of Mathematics \\ Cornell University \\ Ithaca, NY, U.S.A.
}

karola@math. cornell.edu

\author{
Avery St. Dizier ${ }^{\dagger}$ \\ Department of Mathematics \\ University of Illinois at Urbana-Champaign \\ Urbana, IL, U.S.A. \\ stdizie2@illinois.edu
}

\author{
Arthur Tanjaya \\ Department of Mathematics \\ Cornell University \\ Ithaca, NY, U.S.A. \\ amt333@cornell.edu
}

Submitted: May 29, 2021; Accepted: Oct 25, 2021; Published: Nov 5, 2021

(c) The authors. Released under the CC BY-ND license (International 4.0).

\begin{abstract}
Schur polynomials are special cases of Schubert polynomials, which in turn are special cases of dual characters of flagged Weyl modules. The principal specialization of Schur and Schubert polynomials has a long history, with Macdonald famously expressing the principal specialization of any Schubert polynomial in terms of reduced words. We prove a lower bound on the principal specialization of dual characters of flagged Weyl modules. Our result yields an alternative proof of a conjecture of Stanley about the principal specialization of Schubert polynomials, originally proved by Weigandt.
\end{abstract}

Mathematics Subject Classifications: 05E05, 05E10

\section{Introduction}

Schubert polynomials $\mathfrak{S}_{w}$ were introduced by Lascoux and Schützenberger in [12] as distinguished polynomial representatives for the cohomology classes of Schubert cycles in the flag variety. Schubert polynomials generalize Schur polynomials, a classical basis of the ring of symmetric functions.

\footnotetext{
*Supported by CAREER NSF Grant DMS-1847284.

${ }^{\dagger}$ Supported by NSF Grant DMS-2002079.
} 
The principal specialization of Schur polynomials has a long history: $s_{\lambda}(1, \ldots, 1)$ counts the number of semistandard Young tableaux of shape $\lambda$, a number famously enumerated by the hook-content formula, see for instance [22]. Macdonald [14, Eq. 6.11] famously expressed the principal specialization $\mathfrak{S}_{w}(1, \ldots, 1)$ of the Schubert polynomial $\mathfrak{S}_{w}$ in terms of the reduced words of the permutation $w$. Fomin and Kirillov [8] placed this expression in the context of plane partitions for dominant permutations, while after two decades Billey et al. [2] provided a combinatorial proof. Principal specialization of Schubert polynomials has inspired a flurry of recent interest $[9,16,17,19,23,24]$. A major catalyst for the current line of study into $\mathfrak{S}_{w}(1, \ldots, 1)$ is the following result of Weigandt, which generalizes a conjecture of Stanley ([23, Conjecture 4.1]).

Theorem 1 ([24, Theorem 1.1]). For any permutation $w \in S_{n}$,

$$
\mathfrak{S}_{w}(1, \ldots, 1) \geqslant 1+p_{132}(w),
$$

where $p_{132}(w)$ is the number of 132-patterns in $w$.

Weigandt's proof of Theorem 1 works by exploiting the structure of pipe dreams, one of the earliest combinatorial models for Schubert polynomials $[1,7]$. We give an alternative proof of Theorem 1 by generalizing its statement to the setting of dual characters of flagged Weyl modules of diagrams:

Theorem 2. For any diagram D, the dual character $\chi_{D}$ of the flagged Weyl module of D satisfies

$$
\chi_{D}(1, \ldots, 1) \geqslant \operatorname{rank}(D)+1 .
$$

We show in Corollary 19 that Theorem 2 specializes to Theorem 1. Additionally, Theorem 2 implies an analogous result for key polynomials (Corollary 20).

\section{Outline of this paper}

In Section 2 we define dual characters of flagged Weyl modules of diagrams, and we provide necessary background. In Section 3, we define the rank of a diagram and prove Theorem 2. We characterize the case of equality in Theorem 2 and connect to zero-one polynomials. We conclude in Section 4 by describing a simple upper bound version of Theorem 2, and conjecturing a characterization for when equality holds.

\section{Background}

We first define flagged Weyl modules and their dual characters. We then recall the definition of Schubert polynomials and the connection between Schubert polynomials and dual characters. The exposition of this section follows that of [6].

By a diagram, we mean a sequence $D=\left(C_{1}, C_{2}, \ldots, C_{n}\right)$ of finite subsets of $[n]$, called the columns of $D$. We interchangeably think of $D \subseteq[n] \times[n]$ as a collection of boxes $(i, j)$ in a grid, viewing an element $i \in C_{j}$ as a box in row $i$ and column $j$ of the grid. When 
we draw diagrams, we read the indices as in a matrix: $i$ increases top-to-bottom and $j$ increases left-to-right.

Let $G=\operatorname{GL}(n, \mathbb{C})$ be the group of $n \times n$ invertible matrices over $\mathbb{C}$ and $B$ be the subgroup of $G$ consisting of the $n \times n$ upper-triangular matrices. The flagged Weyl module is a representation of $B$ associated to a diagram $D$. The flagged Weyl module of $D$ will be denoted by $\mathcal{M}_{D}$. We will use the construction of $\mathcal{M}_{D}$ in terms of determinants given in [15].

Denote by $Y$ the $n \times n$ matrix with indeterminates $y_{i j}$ in the upper-triangular positions and zeros elsewhere. Let $\mathbb{C}[Y]$ be the polynomial ring in the indeterminates $\left\{y_{i j}\right\}_{i \leqslant j}$. Note that $B$ acts on $\mathbb{C}[Y]$ on the right via left translation: if $f(Y) \in \mathbb{C}[Y]$, then a matrix $b \in B$ acts on $f$ by $f(Y) \cdot b=f\left(b^{-1} Y\right)$. For any $R, S \subseteq[n]$, let $Y_{S}^{R}$ be the submatrix of $Y$ obtained by restricting to rows $R$ and columns $S$.

For $R, S \subseteq[n]$, we say $R \leqslant S$ if $\# R=\# S$ and the $k$ th least element of $R$ does not exceed the $k$ th least element of $S$ for each $k$. For any diagrams $C=\left(C_{1}, \ldots, C_{n}\right)$ and $D=\left(D_{1}, \ldots, D_{n}\right)$, we say $C \leqslant D$ if $C_{j} \leqslant D_{j}$ for all $j \in[n]$.

Definition 3. For a diagram $D=\left(D_{1}, \ldots, D_{n}\right)$, the flagged Weyl module $\mathcal{M}_{D}$ is defined by

$$
\mathcal{M}_{D}=\operatorname{Span}_{\mathbb{C}}\left\{\prod_{j=1}^{n} \operatorname{det}\left(Y_{D_{j}}^{C_{j}}\right) \mid C \leqslant D\right\} .
$$

$\mathcal{M}_{D}$ is a $B$-module with the action inherited from the action of $B$ on $\mathbb{C}[Y]$.

Note that since $Y$ is upper-triangular, the condition $C \leqslant D$ is technically unnecessary since $\operatorname{det}\left(Y_{D_{j}}^{C_{j}}\right)=0$ unless $C_{j} \leqslant D_{j}$. Conversely, if $C_{j} \leqslant D_{j}$, then $\operatorname{det}\left(Y_{D_{j}}^{C_{j}}\right) \neq 0$.

For any $B$-module $N$, the character of $N$ is defined by

$$
\operatorname{char}(N)\left(x_{1}, \ldots, x_{n}\right)=\operatorname{tr}(X: N \rightarrow N)
$$

where $X$ is the diagonal matrix with diagonal entries $x_{1}, \ldots, x_{n}$, and $X$ is viewed as a linear map from $N$ to $N$ via the $B$-action. Define the dual character of $N$ to be the character of the dual module $N^{*}$ :

$$
\begin{aligned}
\operatorname{char}^{*}(N)\left(x_{1}, \ldots, x_{n}\right) & =\operatorname{tr}\left(X: N^{*} \rightarrow N^{*}\right) \\
& =\operatorname{char}(N)\left(x_{1}^{-1}, \ldots, x_{n}^{-1}\right) .
\end{aligned}
$$

Definition 4. For a diagram $D \subseteq[n] \times[n]$, let $\chi_{D}=\chi_{D}\left(x_{1}, \ldots, x_{n}\right)$ be the dual character

$$
\chi_{D}=\operatorname{char}^{*} \mathcal{M}_{D}
$$

Example 5. Let $D$ be the diagram

$$
D=(\{1,3\},\{2,3\})=\begin{array}{|l|l|}
\hline & \\
\hline & \\
\hline & \\
\hline
\end{array}
$$


Then the diagrams $C$ with $C \leqslant D$ are
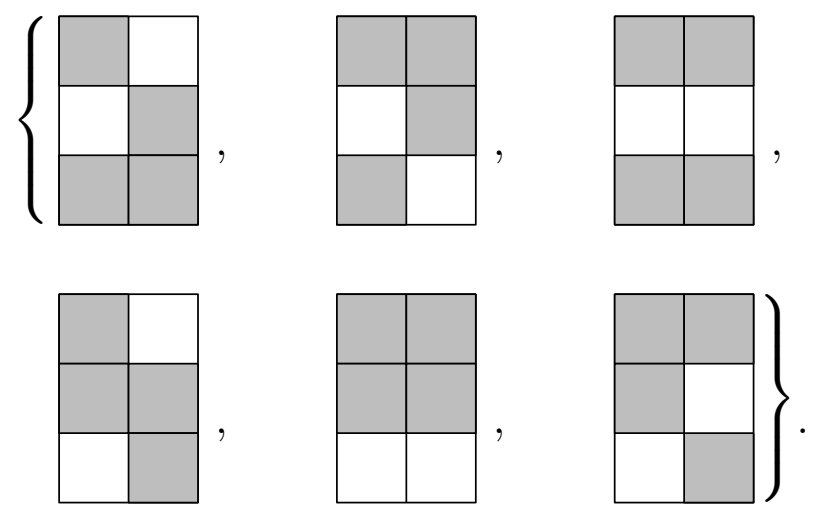

The corresponding products of determinants are

$$
\begin{array}{rlr}
y_{11} y_{22} y_{33}^{2}, & y_{11} y_{12} y_{23} y_{33}-y_{11} y_{13} y_{22} y_{33}, \quad y_{11} y_{12} y_{33}^{2}, \\
y_{11} y_{22} y_{23} y_{33}, & y_{11} y_{12} y_{23}^{2}-y_{11} y_{13} y_{22} y_{23}, \quad y_{11} y_{12} y_{23} y_{33} .
\end{array}
$$

These determinants are all linearly independent eigenvectors of $X$, so

$$
\chi_{D}\left(x_{1}, x_{2}, x_{3}\right)=x_{1} x_{2} x_{3}^{2}+2 x_{1}^{2} x_{2} x_{3}+x_{1}^{2} x_{3}^{2}+x_{1} x_{2}^{2} x_{3}+x_{1}^{2} x_{2}^{2} .
$$

Definition 6. For any diagram $D \subseteq[n] \times[n]$ with columns $D_{1}, \ldots, D_{n}$, we write $x^{D}$ for the monomial

$$
x^{D}=\prod_{j=1}^{n} \prod_{i \in D_{j}} x_{i}
$$

The following two easy results describe the supports and coefficients of dual characters of diagrams.

Theorem 7 (cf. [5, Theorem 7]). For any diagram $D \subseteq[n] \times[n]$, the monomials appearing in $\chi_{D}$ are exactly

$$
\left\{x^{C} \mid C \leqslant D\right\}
$$

Corollary 8. Let $D \subseteq[n] \times[n]$ be a diagram. Fix any diagram $C^{(1)} \leqslant D$ and set $\boldsymbol{m}=x^{C^{(1)}}$. Suppose $C^{(1)}, \ldots, C^{(r)}$ are all the diagrams $C \leqslant D$ such that $x^{C}=\boldsymbol{m}$. Then, the coefficient of $\boldsymbol{m}$ in $\chi_{D}$ is equal to the number of linearly independent polynomials over $\mathbb{C}$ among $\left\{\prod_{j=1}^{n} \operatorname{det}\left(Y_{D_{j}}^{C_{j}^{(i)}}\right) \mid i \in[r]\right\}$.

\subsection{Schubert Polynomials}

Recall the divided difference operators $\partial_{j}$ for $j \in[n-1]$ are operators on the polynomial ring $\mathbb{C}\left[x_{1}, \ldots, x_{n}\right]$ defined by

$$
\partial_{j}(f)=\frac{f-\left(s_{j} \cdot f\right)}{x_{j}-x_{j+1}}=\frac{f\left(x_{1}, \ldots, x_{n}\right)-f\left(x_{1}, \ldots, x_{j+1}, x_{j}, \ldots, x_{n}\right)}{x_{j}-x_{j+1}} .
$$


Definition 9. The Schubert polynomial $\mathfrak{S}_{w}$ of $w \in S_{n}$ is defined recursively on the weak Bruhat order. Let $w_{0}=n n-1 \cdots 21 \in S_{n}$, the longest permutation in $S_{n}$. If $w \neq w_{0}$ then there is $j \in[n-1]$ with $w(j)<w(j+1)$ (called an ascent of $w$ ). The polynomial $\mathfrak{S}_{w}$ is defined by

$$
\mathfrak{S}_{w}= \begin{cases}x_{1}^{n-1} x_{2}^{n-2} \cdots x_{n-1} & \text { if } w=w_{0}, \\ \partial_{j} \mathfrak{S}_{w s_{j}} & \text { if } w(j)<w(j+1) .\end{cases}
$$

Definition 10. The Rothe diagram $D(w)$ of a permutation $w \in S_{n}$ is the diagram

$$
D(w)=\left\{(i, j) \in[n] \times[n] \mid i<w^{-1}(j) \text { and } j<w(i)\right\} .
$$

The diagram $D(w)$ can be visualized as the set of boxes left in the $n \times n$ grid after you cross out all boxes weakly below $(i, w(i))$ in the same column, or weakly right of $(i, w(i))$ in the same row for each $i \in[n]$.

Example 11. If $w=31542$, then

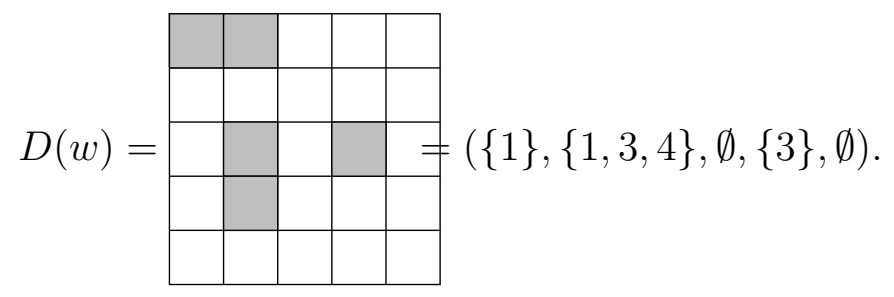

The Schubert polynomial of $w$ is computed by

$$
\mathfrak{S}_{w}=\partial_{2} \partial_{1} \partial_{3} \partial_{2} \partial_{4}\left(x_{1}^{4} x_{2}^{3} x_{3}^{2} x_{4}\right) .
$$

Via Rothe diagrams, Schubert polynomials occur as special cases of dual characters of flagged Weyl modules:

Theorem 12 ([11]). Let $w$ be a permutation with Rothe diagram $D(w)$. Then,

$$
\mathfrak{S}_{w}=\chi_{D(w)} .
$$

\subsection{Key Polynomials}

Key polynomials were first introduced by Demazure for Weyl groups [3], and studied in the context of the symmetric group by Lascoux and Schützenberger in $[12,13]$. Recall the key polynomial $\kappa_{\alpha}$ of a composition $\alpha=\left(\alpha_{1}, \alpha_{2}, \ldots\right)$ is defined as follows. When $\alpha$ is a partition, $\kappa_{\alpha}=x^{\alpha}$. Otherwise, suppose $\alpha_{i}<\alpha_{i+1}$ for some $i$. Then

$$
\kappa_{\alpha}=\partial_{i}\left(x_{i} \kappa_{\beta}\right), \text { where } \beta=\left(\alpha_{1}, \ldots, \alpha_{i+1}, \alpha_{i}, \ldots\right) .
$$

Definition 13 ([10,18]). Fix a composition $\alpha$, and set

$$
l=\max \left\{i \mid \alpha_{i} \neq 0\right\} \quad \text { and } n=\max \left\{l, \alpha_{1}, \ldots, \alpha_{l}\right\} .
$$

The skyline diagram of $\alpha$ is the diagram $D(\alpha) \subseteq[n] \times[n]$ containing the leftmost $\alpha_{i}$ boxes in row $i$ for each $i \in[n]$. 
Example 14. If $\alpha=(3,2,0,1,1)$, then

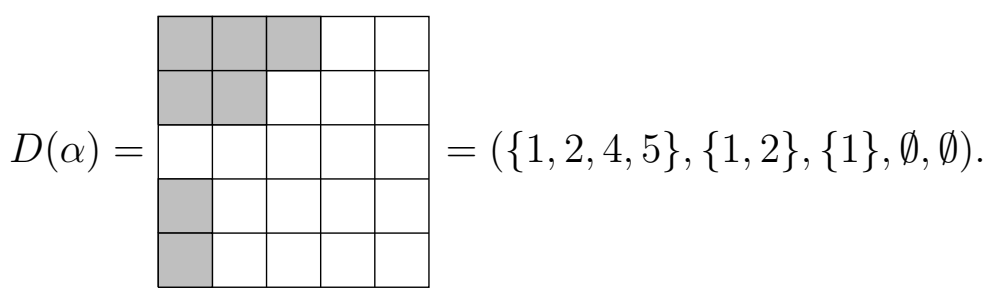

The key polynomial of $\alpha$ is computed by

$$
\kappa_{\alpha}=\partial_{3}\left(x_{3} \partial_{4}\left(x_{4}\left(x_{1}^{3} x_{2}^{2} x_{3} x_{4}\right)\right)\right) .
$$

Theorem 15 ([4]). Let $\alpha$ be a composition with skyline diagram $D(\alpha)$. Then

$$
\kappa_{\alpha}=\chi_{D(\alpha)}
$$

\section{A Lower Bound for $\chi_{D}(1, \ldots, 1)$}

We prove a lower bound for the principal specialization of the dual character of any diagram. We then specialize this bound to Schubert and key polynomials.

Definition 16. Fix a diagram $D$. For each box $(i, j) \in D$, the rank of that box is

$$
\operatorname{rank}_{D}(i, j)=\#\{k \mid 1 \leqslant k \leqslant i \text { and }(k, j) \notin D\} .
$$

The rank of $D$ is

$$
\operatorname{rank}(D)=\sum_{(i, j) \in D} \operatorname{rank}_{D}(i, j)
$$

Lemma 17. Let $D$ be a diagram and let $r=\operatorname{rank}(D)$. Then there are diagrams $C^{0}, C^{1}, \ldots, C^{r-1}$ such that $C^{0}<C^{1}<\cdots<C^{r-1}<D$ and $\operatorname{rank}\left(C^{k}\right)=k$.

Proof. If $r=0$ then the chain consists of just $D$ and there is nothing to prove. Assume $r>0$. We begin with the case that $D$ has a single nonempty column. Without loss of generality, we may write $D=\left(D_{1}\right)=\left(\left\{a_{1}, \ldots, a_{m}\right\}\right)$. Since $\operatorname{rank}(D)>0, D_{1} \neq$ $\{1, \ldots, m\}$. Let $k$ be the largest integer less than $a_{m}$ such that $k \notin D_{1}$. Choose $i$ so that $a_{i}=k+1$ (which must exist by definition of $k$ ). Define

$$
C_{1}=\left(D_{1} \backslash\left\{a_{i}\right\}\right) \cup\{k\} .
$$

Then $C_{1}<D_{1}$, and $\operatorname{rank}\left(C_{1}\right)=\operatorname{rank}\left(D_{1}\right)-1$. By induction, the result follows whenever $D$ has a single nonempty column. Since

$$
\operatorname{rank}\left(\left(D_{1}, \ldots, D_{n}\right)\right)=\sum_{j \in[n]} \operatorname{rank}\left(\left(D_{j}\right)\right)
$$

the general case follows from the single column case by performing the above construction to one column at a time. 
Recall the inverse lexicographic order on monomials: $x^{a}<_{\text {invlex }} x^{b}$ if there exists $1 \leqslant i \leqslant n$ such that $a_{j}=b_{j}$ for $i+1 \leqslant j \leqslant n$, and $a_{i}<b_{i}$.

Lemma 18. If $C<D$, then $x^{C} \neq x^{D}$.

Proof. Let $C=\left(C_{1}, \ldots, C_{n}\right)$ and $D=\left(D_{1}, \ldots, D_{n}\right)$, so $C_{j} \leqslant D_{j}$ for all $j \in[n]$. Fix a column $j$. Then $C_{j} \leqslant D_{j}$ means we can write $C_{j}=\left\{a_{1}, \ldots, a_{m}\right\}$ and $D_{j}=\left\{b_{1}, \ldots, b_{m}\right\}$ with $a_{i} \leqslant b_{i}$ for all $i \in[m]$. Consequently,

$$
\prod_{i \in C_{j}} x_{i} \leqslant \text { invlex } \prod_{i \in D_{j}} x_{i}
$$

Since $C<D$, we know $C_{j}<D_{j}$ for at least one $j \in[n]$. For any such $j$, we have

$$
\prod_{i \in C_{j}} x_{i}<_{\text {invlex }} \prod_{i \in D_{j}} x_{i}
$$

SO

$$
x^{C}=\prod_{j=1}^{n} \prod_{i \in C_{j}} x_{i}<_{\text {invlex }} \prod_{j=1}^{n} \prod_{i \in D_{j}} x_{i}=x^{D} .
$$

In particular $x^{C} \neq x^{D}$.

We now prove Theorem 2.

Proof of Theorem 2. By Theorem 7,

$$
\chi_{D}(1, \ldots, 1) \geqslant \#\left\{x^{C} \mid C \leqslant D\right\} .
$$

By Lemma 17, there exists a chain of $r=\operatorname{rank}(D)+1$ diagrams $C^{0}<C^{1}<\cdots<C^{r-1}<$ D. Thus, by Lemma 18,

$$
\#\left\{x^{C} \mid C \leqslant D\right\} \geqslant \#\left\{x^{C} \mid C \in\left\{C^{0}, C^{1}, \ldots, C^{r-1}, D\right\}\right\}=r+1=\operatorname{rank}(D)+1 .
$$

By specializing Theorem 2 to Rothe diagrams, we obtain a new proof of Theorem 1:

Corollary 19 ([24, Theorem 1.1]). For any permutation $w \in S_{n}$,

$$
\mathfrak{S}_{w}(1, \ldots, 1) \geqslant 1+p_{132}(w),
$$

where $p_{132}(w)$ is the number of 132-patterns in $w$.

Proof. It is enough to show that $p_{132}(w)=\operatorname{rank}(D(w))$. By viewing 132-patterns of $w$ graphically in $D(w)$, one easily observes that 132-patterns are in transparent bijection with tuples $(i, j, k)$ such that $(i, j) \in D(w), 1 \leqslant k<i$, and $(k, j) \notin D(w)$. The quantity $\operatorname{rank}(D(w))$ exactly counts these tuples.

By specializing Theorem 2 to skyline diagrams, we obtain an analogous result for key polynomials. For a composition $\alpha$, let $\operatorname{rinv}(\alpha)$ denote the set of right inversions of $\alpha$, the pairs $i<j$ such that $\alpha_{i}<\alpha_{j}$. 
Corollary 20. For any composition $\alpha$,

$$
\kappa_{\alpha}(1, \ldots, 1) \geqslant 1+\sum_{(i, j) \in \operatorname{rinv}(\alpha)}\left(\alpha_{j}-\alpha_{i}\right) .
$$

We now characterize the case of equality in Theorem 2 .

Definition 21. Let $D$ be any diagram. A pair of boxes $(i, j),\left(i^{\prime}, j^{\prime}\right) \in D$ is called an unstable pair if

- $\operatorname{rank}_{D}(i, j) \geqslant 1$

- $\operatorname{rank}_{D}\left(i^{\prime}, j^{\prime}\right) \geqslant 1$;

- If $i=i^{\prime}$ or $j=j^{\prime}$, then $\operatorname{rank}_{D}(i, j)+\operatorname{rank}_{D}\left(i^{\prime}, j^{\prime}\right) \geqslant 3$.

Proposition 22. A diagram $D$ satisfies $\chi_{D}(1, \ldots, 1)=\operatorname{rank}(D)+1$ if and only if $D$ does not contain an unstable pair.

Proof. Suppose $D$ contains an unstable pair $\left\{(i, j),\left(i^{\prime}, j^{\prime}\right)\right\}$. A simple case analysis shows one can move boxes in $D$ upwards to create diagrams $C, C^{\prime} \leqslant D$ of the same rank with $x^{C} \neq x^{C^{\prime}}$. This implies $\chi_{D}(1, \ldots, 1) \neq \operatorname{rank}(D)+1$.

Assume $D$ contains no unstable pair. If $\operatorname{rank}(D)=0$, then the result follows. Pick $(i, j) \in D$ with $\operatorname{rank}_{D}(i, j) \geqslant 1$. If $\operatorname{rank}_{D}(i, j)>1$, then any other positive rank box would form an unstable pair with $(i, j)$. Hence $(i, j)$ is the only positive rank box of $D$, and the result follows easily.

Suppose $\operatorname{rank}_{D}(i, j)=1$. To avoid unstable pairs, all other positive rank boxes of $D$ either lie in row $i$, or they all lie in column $j$. In either case, they must all have rank exactly 1. If all positive rank boxes of $D$ lie in column $i$, then one observes there is a unique diagram $C \leqslant D$ with rank $k$ for each $k=0,1, \ldots, \operatorname{rank}(D)$, implying the result.

If all positive rank boxes of $D$ lie in row $j$, then one observes that all diagrams $C \leqslant D$ of a fixed rank have the same monomial $x^{C}$, and their determinants span an eigenspace of dimension one in the flagged Weyl module.

We now relate equality in Theorem 2 with the question of when $\chi_{D}$ is zero-one. Recall a polynomial $f$ is called zero-one if all nonzero coefficients in $f$ equal 1.

Proposition 23. If a diagram $D$ satisfies $\chi_{D}(1, \ldots, 1)=\operatorname{rank}(D)+1$, then $\chi_{D}$ is zeroone.

Proof. In order for $\chi_{D}(1, \ldots, 1)=\operatorname{rank}(D)+1$, it must happen that all diagrams $C \leqslant D$ with a fixed rank induce the same monomial $x^{C}$ and have dependent determinants in the flagged Weyl module. Since all diagrams $C, C^{\prime} \leqslant D$ with $x^{C}=x^{C^{\prime}}$ must have the same rank, it follows that all eigenspaces in the flagged Weyl module of $D$ have dimension one. 
We now provide a conjectural characterization of diagrams $D$ such that $\chi_{D}$ is zero-one. Consider the six box configurations shown in Figure 1. In each configuration, an $\times$ (red) indicates the absence of a box; a shaded square (gray) indicates the presence of a box; and an unshaded square (white) indicates no restriction on the presence or absence of a box.

Definition 24. Let $D$ be any diagram. We say $D$ contains a multiplicitous configuration if there are $r_{1}<r_{2}<r_{3}<r_{4}$ and $c_{1}<c_{2}$ so that $D$ restricted to rows $\left\{r_{1}, r_{2}, r_{3}, r_{4}\right\}$ and columns $\left\{c_{1}, c_{2}\right\}$ equals one of the configurations shown in Figure 1, up to possibly swapping the order of the columns.
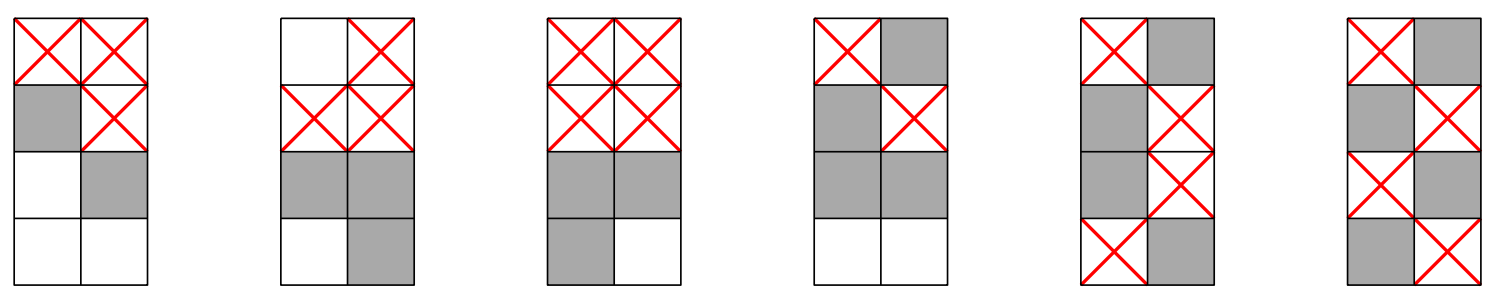

Figure 1: The six multiplicitous configurations.

Example 25. Consider the diagrams $D$ and $D^{\prime}$ shown in Figure 2. The diagram $D$ does not contain instances of any multiplicitous configurations. The diagram $D^{\prime}$ contains instances of each of the multiplicitous configurations.
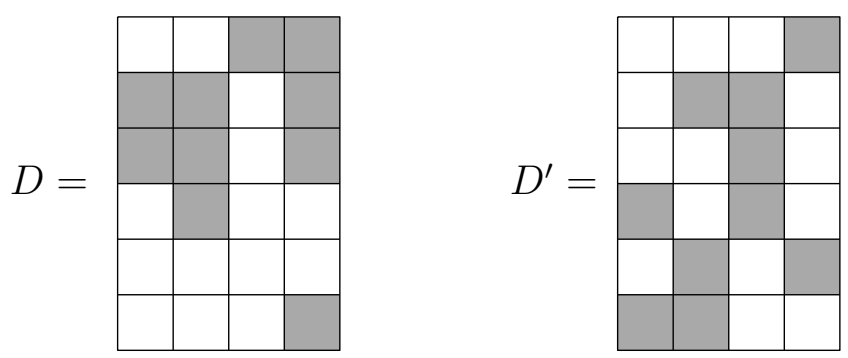

Figure 2:

Proposition 26. If a diagram $D$ contains a multiplicitous configuration, then $\chi_{D}$ is not zero-one.

Proof. It follows immediately from [6, Theorem 5.8] that if the restriction of a diagram $D$ to rows $\left\{i_{1}, \ldots i_{p}\right\}$ and columns $\left\{j_{1}, \ldots, j_{q}\right\}$ equals a diagram $D^{\prime}$, then the largest coefficient appearing in $\chi_{D}$ is bounded below by the largest coefficient appearing in $\chi_{D^{\prime}}$. One can check that the dual characters of each of the multiplicitous configurations are not zero-one. 
Conjecture 27. If $D$ is a diagram such that $\chi_{D}$ is not zero-one, then $D$ contains a multiplicitous configuration.

Together, Proposition 26 and Conjecture 27 specialize to known results for Schubert and key polynomials: [6, Theorem 1] when $D$ is the Rothe diagram of a permutation, and [21, Theorem 1.1] when $D$ is the skyline diagram of a composition.

\section{An Upper Bound for $\chi_{D}(1, \ldots, 1)$}

We recall a trivial upper bound for the principal specialization of the dual character of any diagram. We make a conjecture for the case of equality. From Corollary 8 , it follows immediately that if $c_{\alpha}$ is the coefficient of $x^{\alpha}$ in $\chi_{D}$, then

$$
c_{\alpha} \leqslant \#\left\{C \leqslant D \mid x^{C}=x^{\alpha}\right\} .
$$

In particular,

$$
\chi_{D}(1, \ldots, 1) \leqslant \#\{C \mid C \leqslant D\} .
$$

Fan and Guo gave the following characterization for equality when the diagram $D$ is northwest. Recall a diagram $D$ is northwest if whenever $(i, j),\left(i^{\prime}, j^{\prime}\right) \in D$ with $i>i^{\prime}$ and $j<j^{\prime}$, one has $\left(i^{\prime}, j\right) \in D$.

Theorem 28 ([20]). For any northwest diagram D,

$$
\chi_{D}(1, \ldots, 1)=\#\{C \mid C \leqslant D\}
$$

if and only if D contains no instance of the configuration shown in Figure 3.

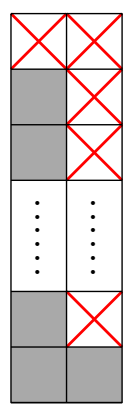

Figure 3:

We conjecturally extend Theorem 28 to all diagrams.

Conjecture 29. Let $D$ be any diagram. Then $\chi_{D}(1, \ldots, 1)=\#\{C \mid C \leqslant D\}$ if and only if $D$ contains no instance of the configuration shown in Figure 4. 


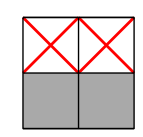

Figure 4:

\section{References}

[1] N. Bergeron and S. Billey. RC-graphs and Schubert polynomials. Experiment. Math., 2(4):257-269, 1993.

[2] S. Billey, A. Holroyd, and B. Young. A bijective proof of Macdonald's reduced word formula. Algebr. Comb., 2(2):217-248, 2019.

[3] M. Demazure. Key polynomials and a flagged Littlewood-Richardson rule. Bull. Sci. Math., 98:163-172, 1974.

[4] M. Demazure. Une nouvelle formule des caracteres. J. Combin. Theory Ser. A, 70(1):107-143, 1995.

[5] A. Fink, K. Mészáros, and A. St. Dizier. Schubert polynomials as integer point transforms of generalized permutahedra. Adv. Math., 332:465-475, 2018.

[6] A. Fink, K. Mészáros, and A. St. Dizier. Zero-one Schubert polynomials. Math. Z., 2020 .

[7] S. Fomin and A.N. Kirillov. The Yang-Baxter equation, symmetric functions, and Schubert polynomials. Discrete Math., 153(1):123-143, 1996. Proceedings of the 5th Conference on Formal Power Series and Algebraic Combinatorics.

[8] S. Fomin and A.N. Kirillov. Reduced words and plane partitions. J. Algebraic Combin., 6(4):311-319, 1997.

[9] Y. Gao. Principal specializations of Schubert polynomials and pattern containment. European J. Combin., 94:103291, 2021.

[10] J. Haglund, M. Haiman, and N. Loehr. A combinatorial formula for nonsymmetric Macdonald polynomials. American Journal of Mathematics, 130(2):359-383, 2008.

[11] W. Kraśkiewicz and P. Pragacz. Foncteurs de Schubert. C. R. Acad. Sci. Paris Sér. I Math., 304(9):209-211, 1987.

[12] A. Lascoux and M.-P. Schützenberger. Polynômes de Schubert. C. R. Acad. Sci. Paris Sér. I Math., 294(13):447-450, 1982.

[13] A. Lascoux and M.-P Schützenberger. Structure de Hopf de l'anneau de cohomologie et de l'anneau de Grothendieck d'une variété de drapeaux. C. R. Acad. Sci. Paris Sér. I Math., 295(11):629-633, 1982.

[14] I. Macdonald. Notes on Schubert polynomials. Publ. LaCIM, UQAM, Montrèal, 1991.

[15] P. Magyar. Schubert polynomials and Bott-Samelson varieties. Comment. Math. Helv., 73(4):603-636, 1998.

[16] G. Merzon and E. Smirnov. Determinantal identities for flagged Schur and Schubert polynomials. Eur. J. Math., 2:227-245, 2016. 
[17] K. Mészáros and A. Tanjaya. Inclusion-exclusion on Schubert polynomials, 2021. arXiv:2102.11179.

[18] C. Monical, N. Tokcan, and A. Yong. Newton polytopes in algebraic combinatorics. Selecta Math. (N.S.), 25(66), 2019.

[19] A.H. Morales, I. Pak, and G. Panova. Asymptotics of principal evaluations of Schubert polynomials for layered permutations. Proc. Amer. Math. Soc., 147(4):13771389, 2019.

[20] N.J. Fan and P.L. Guo. Upper bounds of Schubert polynomials, 2020. Sci. China Math., 2021.

[21] R. Hodges and A. Yong. Multiplicity-free key polynomials, 2020. arXiv:2007.09229.

[22] R.P. Stanley. Enumerative Combinatorics, volume 2 of Cambridge Studies in Advanced Mathematics. Cambridge University Press, 1999.

[23] R.P. Stanley. Some Schubert shenanigans, 2017. arXiv:1704.00851.

[24] A. Weigandt. Schubert polynomials, 132-patterns, and Stanley's conjecture. Algebr. Comb., 1(4):415-423, 2018. 